\title{
Effects of Mother and Father Dominance on Offspring Sex in Contemporary Humans
}

\author{
Jaime L. Palmer-Hague ${ }^{1,2} \cdot$ Neil V. Watson ${ }^{1}$
}

Received: 24 June 2015 / Revised: 29 July 2015 / Accepted: 5 August 2015 /

Published online: 18 August 2015

C) Springer International Publishing 2015

\begin{abstract}
Variation in human sex ratios has been the focus of empirical investigation for decades, but no studies have examined the relationship between parental mating behavior and future offspring sex. Here we tested whether dominance cues in the faces and behavior of mothers and fathers were associated with the sex of their future offspring. Our results show that maternal facial cues indicating lower dominance status may be associated with increased probability of a daughter as a first-born child, but only when moderated by high paternal facial dominance. We also show that both mother and father's dominance behavior increases the probability of a first-born son. These results suggest that the effect of maternal dominance on the sex of offspring depends on the dominance of their male partner; however, future research should investigate additional characteristics that influence long-term mating decisions in women according to future offspring sex.
\end{abstract}

Keywords Trivers-Willard hypothesis · Maternal dominance hypothesis · Dominance Sex ratio $\cdot$ Mate selection $\cdot$ Faces

\section{Introduction}

Despite considerable empirical interest in human mating behavior, very little attention has been paid to the potential relationship between male and female mate preferences for dominance and subsequent offspring sex ratio. Although there is a large body of literature implicating physical and behavioral indicators of dominance as important for

Jaime L. Palmer-Hague

jaime.palmerhague@twu.ca

1 Department of Psychology, Simon Fraser University, 8888 University Drive, Burnaby, BC V5A 1S6, Canada

2 Department of Psychology, Trinity Western University, 7600 Glover Road, Langley, BC V2Y 1Y1, Canada 
mate choice, no studies have investigated whether women's preferences for dominance may be fundamentally related to the sexes of her future offspring. Here we examine whether facial and behavioral dominance in men and women is related to facial and behavioral dominance in their mates, and whether or not such characteristics reliably predict their tendency to produce sons compared to daughters. These possibilities have not been investigated previously.

\section{Offspring Sex Ratio Variation}

Despite strong and pervasive preferences for male offspring that alter sex ratios in some countries (reviewed in Dahl and Moretti 2008; Hank 2007; reviewed in Hesketh and Xing 2006; Marleau and Saucier 2002; Ray et al. 2012), in most others, the human sex ratio at birth remains virtually unchanged at approximately 105-107 males to 100 females (e.g., Central Intelligence Agency 2013). Interestingly, though, many instances of variations in this sex ratio in response to natural factors have been reported. For example, increases in male births have been reported following major wars, in response to environmental toxins, in parents experiencing stress and illness, in parents who have been married for a shorter time, and when conception occurs at the end of a woman's ovulation period (reviewed in James 1996, 2004, 2009, 2010). Although the underlying mechanisms for these changes in sex ratio have not yet been definitively established, parental ability to vary the sexes of their offspring in response to dynamic environmental influences would effectively enhance their fitness.

The Trivers-Willard Hypothesis (TWH, Trivers and Willard 1973) proposes that parents should maximize their own reproductive success by producing the sex of offspring that will benefit most from their current 'condition'. In other words, if parents are in 'good condition', they should be more likely to produce sons who, through inheritance of this 'good condition', will be more likely to succeed in obtaining mates. Parents in 'poor condition', on the other hand, should be more likely to produce daughters because their reproductive success will be less negatively affected by their daughters' condition. The TWH has been studied in a variety of non-human animals, with mixed results (reviewed in Brown 2001; Clutton-Brock and Iason 1986; Hewison and Gaillard 1999; Hiraiwa-Hasegawa 1993). Sheldon and West (2004) suggested that the lack of consistency is due to excessive focus on physiological characteristics of parents, such as body size and nutrition, rather than on behavioral measures, such as dominance prior to conception.

\section{Dominance and Offspring Sex Ratio}

In humans, a variety of physiological and behavioral characteristics associated with dominance and status, traits that increase male, but not female, reproductive success (reviewed in Campbell 1999; Puts 2010; Puts et al. 2012), have been shown in parents who have more sons. For example, taller and heavier parents have more sons than parents who are shorter and lighter (Kanazawa 2005, but see Gelman 2007 and response in Kanazawa and Reyniers 2009; Manning et al. 1996). It has also been reported that more violent (i.e., aggressive) men have more sons than men who are not violent (Kanazawa 2008). In addition, Gibson and Mace (2003) found that women with greater muscle mass in their upper arms - a proxy for nutritional status (i.e., resource 
acquisition ability) - were significantly more likely to conceive sons than daughters. Parents with lower (i.e., more masculine) ratios of the 2 nd to the 4 th digit of their hands (2D:4D) also have more sons than daughters (Manning et al. 2002). Interestingly, 2D:4D ratios may indicate dominance and masculinity to others (Neave et al. 2003), as well as positively correlate with men's self-reported physical attractiveness (Manning and Quinton 2007). Men who are billionaires, and sons of men who are billionaires, also have more sons than daughters (Cameron and Dalerum 2009). Taken together, these results suggest that dominance and its related characteristics in parents influence the conception of sons in ways that are congruent with the TWH.

It has been proposed that levels of parental hormones at the time of conception could facilitate variation in offspring sex. Characteristics of both parents have been associated with offspring sex, and therefore James (1987) proposed that hormone levels in both parents are responsible for this variation. However, the mechanism by which paternal hormone levels could actually facilitate changes in the probability of a male or female offspring being conceived remains unclear. In contrast, the Maternal Dominance Hypothesis (MDH; Grant 1998) proposes that only the woman's endogenous testosterone level at the time of conception facilitates the production of male offspring. According to the $\mathrm{MDH}$, more dominant women have higher testosterone, which makes their ova more receptive to Y-chromosome bearing sperm. Importantly, Grant proposes that this dominant behavior is fundamentally important for enhancing the reproductive success of her offspring; thus, to some degree, her sons eventually display dominant behavior in response to her interactions with them and others. By producing a son high in quality (i.e., with potential for high dominance), rather than a daughter whose quality will remain unaffected, she therefore enhances her own fitness.

Interestingly, both Grant (1990, 1994) and Akande (1999) have shown that women who scored higher in dominance were more likely to give birth to sons than daughters. In addition, women with higher testosterone levels scored higher in dominance (Grant and France 2001), and male embryos were more likely than female embryos to develop from bovine ova contained in follicles with elevated fluid testosterone concentrations (Grant and Irwin 2005). Because dominance in women can be both stable (i.e., a trait), and variable depending on environment and context (i.e., state), the MDH also explains how the probability of a given woman having either a male or female offspring can vary for different conceptions. Thus, maternal dominance may have an important influence on female reproductive outcomes.

Maternal dominance behavior could influence her male offspring through modeling or epigenetic mechanisms, as Grant (1998) has suggested, but it is also possible that mothers who are more likely to produce sons seek out mates who can either effectively contribute directly (i.e., through contribution of genes), indirectly (i.e., through modeling behavior), or both to the development of dominance behavior in her sons. In other words, in accordance with TWH, women might enhance their fitness through pairing with men who are most likely to pass reproductive benefits to their offspring. Women's preference for men's dominance in this context would also explain why much of the research to date has shown that characteristics of both parents are associated with offspring sex. This possibility has not yet been tested. 


\section{Dominance, Mating Behavior, and Mate Preferences in Men and Women}

Women place a high value on dominance and status in potential mates (reviewed in Buss 2007; reviewed in Cashdan 1998; Sadalla et al. 1987), making it a particularly important characteristic for male reproductive success. Indeed, sexual selection has led to various male traits that serve as cues to these characteristics, one of which is the face. Mueller and Mazur (1997) showed that perceived dominance from facial photographs of military cadets significantly predicted their final career rank as well as the number of children and grandchildren they produced. Similarly, Fink et al. (2007) found that women rated the faces of physically stronger men to be more attractive, dominant, and masculine than less physically strong men. Studies have also shown that ratings of men's physical strength from their faces accurately predicted their actual upper body strength (Sell et al. 2009). More recently, researchers have shown that men's facial structure is positively associated with their perceived and demonstrated aggressive behavior (Carré and McCormick 2008; Lefevre et al. 2014; but see Özener 2012) as well as their fighting ability and perceived formidability (Trebicky et al. 2013; Zilioli et al. 2014), suggesting that it serves as an effective cue to their dominance behavior.

Although most women prefer dominant mates, there is some evidence this is not true for all women in all circumstances. For example, studies of female preferences for masculinity, serving as a signal of male dominance (e.g., Boothroyd et al. 2007; Fink et al. 2007), have yielded mixed results. Whereas several studies have found that women prefer a more masculine face to a less masculine face (Burriss et al. 2011; DeBruine et al. 2006; Johnston et al. 2001), others have shown the opposite pattern (Perrett et al. 1998; Rhodes et al. 2000), or no preference (Scott et al. 2010) (reviewed in Rhodes 2006). Although high conception-risk, greater self-perceived and other-rated attractiveness, and focus on a short-term relationship explain factors involved in female preferences for more masculine mates (reviewed in Puts et al. 2012), individual differences in preferences for masculinity and dominance, particularly for a long-term partner, remain unresolved.

One possible explanation for variability in women's preferences for dominance is that they are influenced by her tendency to produce male or female offspring. Women who are more likely to conceive sons might prefer characteristics that would most benefit their sons in reproduction, such as those that signal dominance, status, and ability to provide resources. On the other hand, women who are more likely to produce female offspring, might prefer mates with characteristics that would most benefit their daughters in reproduction, such as physical attractiveness or willingness to invest in parenting. Interestingly, more masculine faces are rated less likely to invest in offspring (Perrett et al. 1998), suggesting that individual differences in women's preferences for masculinity could reflect different priorities being given to genetic quality ('good genes hypothesis', Trivers 1972) compared to parental investment. Internal factors associated with a woman's current tendency to produce male or female offspring, such as endogenous T, may therefore be an important mediator of female mate choice.

Although less is known about women's dominance, several studies have shown that their faces serve as effective cues as well. Sell et al. (2009) showed that even though the effect was not as strong as for men, ratings of physical strength from women's faces positively predicted their actual upper body strength. Similarly, Geniole et al. (2012) and Lefevre et al. (2014) showed that women's facial structure was positively related to 
ratings of aggressiveness by others, and self-reported verbal aggression, respectively. Quist et al. (2011) also showed that viewers rated the faces of women who were higher in self-reported dominance as more masculine; interestingly, though, these women were not rated any more or less attractive, suggesting that men do not use facial dominance as a factor in making mate choice decisions. In fact, other studies have reported similar findings. Males do not tend to exhibit preferences for dominant or high status females (Sadalla et al. 1987; reviewed in Campbell 1999), suggesting that traits of dominance are much more important for male compared to female reproductive success.

\section{Assortative Mating and Offspring Sex Ratio}

Studies in support of the TWH in humans provide support for mate choice based on characteristics associated with offspring sex (e.g., height and weight for males, physical attractiveness for females), but no studies have directly investigated characteristics of dominance in couples. Two lines of research suggest that there may be a relationship. First, women with a lower waist-to-hip ratio (i.e., more feminine and attractive (e.g., Singh 1994)) are reportedly more likely to value the provision of resources by mates than women with a higher waist-to-hip ratio (i.e. more masculine and less attractive) (Pawlowski and Jasienska 2008). Given that women with higher waist measurements have more sons than daughters (Manning et al. 1996; Singh and Zambarno 1997), and that dominance cues in male faces might be considered a signal of lowered willingness to invest in offspring (Perrett et al. 1998), but not necessarily reduced capacity to earn resources, it makes sense that women who are more likely to produce females would prefer less dominant-looking mates. Second, there is some evidence that women prefer masculine faces when their testosterone levels are high compared to when they are low (Welling et al. 2007). Given that testosterone level may be associated with the conception of sons (Grant and France 2001), this also supports the idea that women may prefer more dominant mates when they are more likely to conceive a son. These possibilities have not yet been tested.

\section{Overview of the Present Study}

We investigated the facial and behavioral dominance characteristics of men and women in committed, long term relationships based on the known sexes of their offspring. Specifically, we examined facial ratings of mothers and fathers from photographs taken during their reproductive years, as well as composite measures of behavioral dominance later in life, to test the hypothesis that mothers and fathers who were more dominant, as evidenced by facial ratings and behavioral dominance scores, would be more likely to conceive sons than those who are less dominant.

\section{Materials and Methods}

\section{Parent Participants}

Parents were recruited for the study as part of a larger study involving mate preferences in male and female undergraduates $(N=132)$. They completed demographic and 
behavioral questionnaire packages and provided photographs of themselves. Packages were provided and completed information was returned to the laboratory via their child. A total of 132 mothers and 129 fathers participated. Written informed consent was provided, and the Simon Fraser University Office of Research Ethics approved all study procedures.

\section{Photographs}

Mothers and fathers were asked to provide one photograph of themselves from around the time of their marriage or beginning of their relationship. Given the retrospective nature of the study, as well as concern for maintaining ecological validity, we placed minimal restrictions on the photographs collected and made the assumption that parents would submit photographs that represented an optimal depiction of themselves. Participants were asked to submit photographs where the person was facing forward and not wearing glasses whenever possible. Individual variation in peripheral features such as hairstyle, jewelry, and clothing was permissible. The majority of parents were smiling in their photographs: 79 fathers ( $65 \%$ of total; 36 first-born son, 43 first-born daughter), and 93 mothers (77\% of total; 39 first-born son, 54 first-born daughter). Photographs were collected in digital format via email, or in hardcopy in the laboratory, where they were scanned in to JPG format.

\section{Behavioral Dominance Measures}

Mothers and fathers were asked to independently complete the following three behavioral dominance measures: (1) the Sociable and Aggressive Dominance subscales (SD and AD, Kalma et al. 1993): a 15-item questionnaire that measures interpersonal behaviors related to aggressive dominance (e.g., "I find it important to get my way.", "I can lie without anybody noticing it.") and sociable dominance (e.g., "I have no problems talking in front of a group.", "I certainly have self-confidence.") within groups, respectively; (2) the Dominance subscale of the International Personality Items Pool (IPIP, http://ipip.ori.org/ipip/; Goldberg et al. 2006): a brief, 11-item questionnaire assessing the degree to which a person dominates others in social interactions, and; (3) the Simple Adjective Test (SAT, Grant 1998): a 64-item checklist with 13 target items that measures the subjective ratings of feelings associated with behavioral dominance.

\section{Face Judgments and Ratings}

Rating Participants A total of 79 participants rated parent faces, in return for partial course credit in a psychology class. Data from 6 participants were excluded for the following reasons: misunderstanding task directions $(n=2)$, responses to fewer than $50 \%$ of the faces $(n=1)$, and apparent falsification of answers (e.g., repeated pattern of responses throughout) $(n=3)$. The remaining 73 undergraduate participants -36 males (Mean age $\pm \mathrm{SD}=20.76 \pm 1.87$ years) and 37 females (Mean age $\pm \mathrm{SD}=20.87 \pm 2.49$ ) made judgments of offspring sex and rated masculinity, attractiveness, and dominance in parents' faces. High inter-rater agreement was obtained for masculinity, attractiveness, and dominance for both mothers' and fathers' faces (all ICCs>.90). 
Preparation and Presentation of Stimuli A total of 243 photographs (121 mothers and 122 fathers) were rated. Age at the time the photograph was taken was available for 118 mothers (Mean $\pm \mathrm{SD}=25.46 \pm 4.11$ years) and 120 fathers (Mean $\pm \mathrm{SD}=28.16 \pm$ 5.29 years). Each digital image was cropped to include mostly the participant's face, and re-sized (if necessary) to a consistent height of $12.25 \mathrm{~cm}$ and width of approximately $9 \mathrm{~cm}$. Some hair and clothing was visible in the photographs. Faces were presented one at a time with an identification number underneath each one that corresponded to questions presented in paper and pencil format. Faces were alternated male then female, and presented in 4 blocks of 61 photographs (60 in the final block) with opportunities to take a break between each block. There was no significant effect of block order on any of the ratings for facial masculinity, attractiveness, or dominance, or for accuracy of sex-of-offspring judgments (all $p<.18$ ).

Rating Procedures Participants completed ratings in groups in a departmental computer facility, but were not permitted to communicate during the task. Each participant was seated individually at a computer and presented with the faces for rating. Prior to beginning the ratings, participants were presented with written instructions directing them to rate faces based on their own initial judgments and to focus on faces only, rather than hairstyles, jewelry, clothing, or other material items that may or may not be present in the photographs. No written descriptions or definitions of masculinity, attractiveness, or dominance were provided. Participants were able to view each face for as long as they wanted while they made their judgments. In the rare instance that a participant recognized an individual in a photograph, they were instructed to check a box identifying this, and not to make ratings for that person.

For each face, participants were asked to first predict the sex of child that the person would have as their first-born child as well as to rate the face for masculinity/femininity, attractiveness/unattractiveness, and dominance/submissiveness using 7-point rating scales. Sex of first-born child as opposed to sex-ratio or sex of later-born child was chosen as a response measure because the photographs were taken as close to the beginning of the parents' relationship as possible and thus they provided the best measure of mother and father facial characteristics prior to engaging in reproduction. This allowed for an approximate measure of each parent's preferences at the time of mate choice.

\section{Data Analyses}

Demographic characteristics of parents of first-born sons and first-born daughters were compared using independent-samples t-tests, Mann-Whitney U tests, and chi square tests as appropriate. To determine whether participants could accurately judge sex of first-born offspring from faces, accuracy and response bias in judgments for sex of offspring were calculated for each participant using signal detection theory (Stanislaw and Todorov 1999). If a participant omitted a response, their total accuracy and bias was calculated without including that item. Overall accuracy scores were compared to chance guessing (0.50) using a one-sample $t$-test. Scores were then analyzed using two mixed model ANOVAs (one for fathers, one for mothers) with sex of offspring as the within-subjects variable and sex of rater as the between-subjects variable. 
Facial and behavioral characteristics in mothers and fathers were explored with zeroorder Pearson correlations. For faces, binary logistic regressions were used to determine the relationships between parental dominance and offspring sex using mother dominance, father dominance, and their interaction as predictors, and sex of first-born child as the dependent variable. Mother and father dominance in faces were first corrected for the effects of masculinity, attractiveness, and age at the time the photograph was taken using two initial linear regressions (one for mother, one for father) run with these variables as predictor variables and facial dominance as the dependent variable (see Cornwell and Perrett 2008). This was done for three reasons. First, masculinity, dominance, and attractiveness are all related concepts, and are important in mate selection (reviewed in Puts 2010; Puts et al. 2012). Second, although perceptions of masculinity and dominance were highly correlated for fathers' faces in our sample (Table 2), they may be differently represented in the faces of both men and women (Lefevre et al. 2014), suggesting that each contributes differently to facial appearance. Third, our photos represented a wide range of ages (mothers, 19 years, and fathers, 28 years), and age was negatively correlated with perceived femininity and attractiveness in mothers ( $r=-40, p<.01$ and $r=-.45, p<.01$, respectively). Standardized residuals obtained from these preliminary analyses were then used in the binary logistic regression models. These standardized residuals were also used to assess the effects of sex of first-born child and facial expression (smiling and not smiling) on dominance ratings using two-way ANOVAs (one for mother, one for father).

For dominance behavior, composite z-scores for mother and father dominance were calculated by creating z-scores for each questionnaire, summing the four z-scores for each participant, and then calculating the z-score for the composite. Composite z-scores for mothers, fathers, and their interaction, were then analyzed using binary logistic regression with sex of the first-born offspring as the dependent variable. Sex of firstborn child was chosen to circumvent the strong effect of parent stopping rules (i.e., the decision whether to have an additional child based on the sexes of existing children) on statistical analysis of sex ratios (e.g., Stansfield and Carlton 2007; Gelman 2007). Given the preliminary nature of our study, we sought to minimize Type II error and therefore significance level for all analyses was set to $p \leq .05$.

\section{Results}

\section{Characteristics of Parents}

Parents of a male first-born child $(n=63)$ were well matched with parents of a female first-born child $(n=69)$ in demographic characteristics. There were no significant differences between groups in age, ethnicity, marital status, or length of relationship (all $p>.05$ ). The majority of couples (first-born son, $73 \%$, first-born daughter, $83 \%$ ) had at least 2 children.

\section{Rater Judgments of sex of First-Born Child}

Bias values more than \pm 3 SD from the mean were identified for five participants, suggesting that their judgments were biased towards choosing male or female offspring. 
Data for these participants were excluded. Overall, participants were not significantly better than chance at judging the sexes of people's first-born children (Mean accuracy \pm $\mathrm{SD}=.50 \pm .05, t(67)=.03, p=.98)$. Similarly, there were no significant main effects of sex of parent, sex of rater, or their interaction $(p>.05)$.

\section{Parent Facial Characteristics}

Inter-rater agreement for masculinity, attractiveness, and dominance ratings was high (all ICCs $>.90$ ). We therefore calculated an average for each characteristic for each face. These averages were used for all further analyses of facial characteristics. Descriptive correlations for facial masculinity, attractiveness, and dominance ratings are shown for separately for mothers and fathers in Tables 1 and 2, respectively. Correlations between mother characteristics and father characteristics are shown in Table 3.

Mother and father attractiveness were significantly positively correlated $(r=.27$, $p<.01)$. No significant correlations were found for mother and father facial masculinity $(r=.11, p>.05)$, but there was a trend towards significance for mother and father facial dominance $(r=.16, p=.08)$ (Table 3$)$.

\section{Parent Facial Dominance Characteristics as Predictors of Sex of First-Born}

In order to control for potential effects of masculinity, attractiveness, and age on ratings of facial dominance, we ran initial linear regressions with these variables as predictors and dominance as the dependent variable. Similar approaches have been used in previous research (e.g., Cornwell and Perrett 2008). Standardized residuals obtained from these initial analyses were used for all further analyses.

Because our sample of photographs contained both faces that were smiling (93 mothers, 77 fathers) and not smiling (28 mothers, 42 fathers), we first

Table 1 Zero-order correlations between facial and behavioral characteristics of mothers

\begin{tabular}{|c|c|c|c|c|c|c|c|c|}
\hline & SAT & IPIP & SD & $\mathrm{AD}$ & DOM COMP & Facial Masc. & Facial Att. & Facial Dom. \\
\hline \multicolumn{9}{|l|}{ SAT } \\
\hline DOM & $.27 \dagger$ & & & & & & & \\
\hline SD & $.26 \dagger$ & $.35 \dagger$ & & & & & & \\
\hline $\mathrm{AD}$ & $.24 \dagger$ & $.55 \dagger$ & $.29 \dagger$ & & & & & \\
\hline DOM COMP & $.63 \dagger$ & $.77 \dagger$ & $.67 \dagger$ & $.74 \dagger$ & & & & \\
\hline Facial Masc. & -.12 & -.03 & $<-.01$ & -.11 & -.09 & & & \\
\hline Facial Att. & -.11 & $<.01$ & -.09 & -.08 & -.07 & $.90 \dagger$ & & \\
\hline Facial Dom. & $-.23 *$ & $<-.01$ & $.24 \dagger$ & -.10 & $-.20 *$ & .01 & $.19 *$ & \\
\hline
\end{tabular}

Significant correlations are in boldface

SAT Simple Adjective Test, IPIP Dominance subscale of the IPIP, SD Sociable Dominance subscale, $A D$ Aggressive Dominance subscale, DOM COMP dominance composite z-score, Masc. masculinity, Att. attractiveness, Dom. dominance

$* p<.05 \dagger p<.01$ 
Table 2 Zero-order correlations between facial and behavioral characteristics of fathers

\begin{tabular}{|c|c|c|c|c|c|c|c|c|}
\hline & SAT & DOM & SD & $\mathrm{AD}$ & DOM COMP & Facial Masc. & Facial Att. & Facial Dom. \\
\hline \multicolumn{9}{|l|}{ SAT } \\
\hline DOM & $.28 \dagger$ & & & & & & & \\
\hline SD & $.25 \dagger$ & $.34 \dagger$ & & & & & & \\
\hline $\mathrm{AD}$ & .09 & $.40 \dagger$ & $.21 *$ & & & & & \\
\hline DOM COMP & $.61 \dagger$ & $.76 \dagger$ & $.67 \dagger$ & $.64 \dagger$ & & & & \\
\hline Facial Masc. & -.05 & .05 & .10 & .03 & .05 & & & \\
\hline Facial Att. & -.07 & .02 & .18 & -.09 & .02 & $-.24 \dagger$ & & \\
\hline Facial Dom. & -.11 & -.04 & -.11 & $<.01$ & -.02 & $-.79 \dagger$ & .15 & \\
\hline
\end{tabular}

Significant correlations are in boldface

SAT Simple Adjective Test, DOM Dominance subscale of the IPIP, $S D$ Sociable Dominance subscale, $A D$ Aggressive Dominance subscale, DOM COMP dominance composite z-score, Masc. masculinity, Att. attractiveness, Dom. dominance

$* p<.05 \dagger p<.01$

assessed the influence of facial expression on dominance ratings using two twoway ANOVAs (one for mothers' faces, one for fathers' faces) with sex of firstborn child and facial expression (smiling and not smiling) as the independent variables.

For mothers' faces, there was a significant main effect of sex of first-born $(F(117)=$ $4.66, p=.03$ ), with mothers of a son rated more dominant (Mean $\pm \mathrm{SD}=.06 \pm 1.10)$ than mothers of a daughter (Mean $\pm \mathrm{SD}=-.06 \pm .87$ ). The main effect of facial expression was not significant, but there was a significant interaction effect of sex of first-born child and facial expression $(F(117)=10.70, p<.01)$ (Fig. 1). Follow-up t-tests revealed that

Table 3 Zero-order correlations between facial and behavioral characteristics of mothers and fathers

\begin{tabular}{|c|c|c|c|c|c|c|c|c|c|}
\hline & & \multicolumn{8}{|c|}{ Father } \\
\hline & & SAT & DOM & SD & $\mathrm{AD}$ & DOM COMP & Facial Masc. & Facial Att. & Facial Dom. \\
\hline \multirow[t]{8}{*}{ Mother } & SAT & $.27 *$ & $<.01$ & .04 & .07 & .15 & -.14 & -.10 & .16 \\
\hline & DOM & $.20 *$ & .07 & $.27 \dagger$ & .17 & $.27 \dagger$ & -.04 & $<-.01$ & -.08 \\
\hline & $\mathrm{SD}$ & .10 & .08 & $.21 *$ & .18 & $.22 *$ & .14 & $<.01$ & .07 \\
\hline & $\mathrm{AD}$ & .13 & .02 & .08 & $.24 \dagger$ & .17 & .10 & $-.19 *$ & $>.01$ \\
\hline & DOM COMP & $.25 \dagger$ & .06 & $.21 *$ & $.23 *$ & $.28 \dagger$ & .06 & -.12 & .04 \\
\hline & Facial Masc. & -.14 & -.07 & .02 & $-.24 *$ & -.16 & .11 & $.23 *$ & $-.24 \dagger$ \\
\hline & Facial Att. & -.07 & -.04 & .05 & $-.21 *$ & -.10 & .10 & $.27 \dagger$ & $-.20 *$ \\
\hline & Facial Dom. & $<.01$ & .03 & -.12 & -.16 & -.09 & -.18 & .12 & .16 \\
\hline
\end{tabular}

Significant correlations are in boldface

SAT Simple Adjective Test, DOM Dominance subscale of the IPIP, SD Sociable Dominance subscale, $A D$ Aggressive Dominance subscale, DOM COMP dominance composite z-score, Masc. masculinity, Att. attractiveness, Dom. dominance

$* p<.05 \dagger p<.01$ 
when not smiling, mothers of a son were rated significantly more dominant (Mean \pm $\mathrm{SD}=.73 \pm 1.10)$ than mothers of a daughter $(\mathrm{Mean} \pm \mathrm{SD}=-.43 \pm .98)(t(25)=-2.72$, $p=.01)$. This difference was not significant when mothers were smiling $(p>.05)$.

For fathers' faces, there was a significant main effect of facial expression $(F(118)=$ $20.88, p<.01$ ), with those who were not smiling (Mean $\pm \mathrm{SD}=.52 \pm 1.02$ ) rated more dominant than those who were smiling (Mean $\pm \mathrm{SD}=-.28 \pm .85)$. Neither the main effect of sex of first-born or the interaction effect of sex of first-born and facial expression was significant $(p>.05)$.

In order to assess the relationship of mother facial dominance, father facial dominance, and their interaction to the sex of first-born offspring, we first conducted binary logistic regression analyses using the entire sample of photographs. Results obtained are shown in Table 4. The first model, with only mother dominance and father dominance included as predictors, was not significant $\left(-2 \mathrm{LL}=160.41, \chi^{2}(2, n=\right.$ $\left.117)=1.58, p=.46, R^{2}=.02\right)$. When the interaction between mother and father dominance was added to the model, it approached significance $\left(-2 \mathrm{LL}=154.56, \chi^{2}(3\right.$, $\left.n=117)=7.45, p=.06, R^{2}=.08\right)$ and accurately predicted $72 \%$ of female first-born offspring and $46 \%$ of male first-born offspring, yielding an overall classification rate of $60 \%$.

Given our interest in the interaction between mother and father dominance a priori, we further explored the mother facial dominance $\mathrm{X}$ father facial dominance interaction using simple slopes analyses for binary models (Dawson 2014), which revealed that the

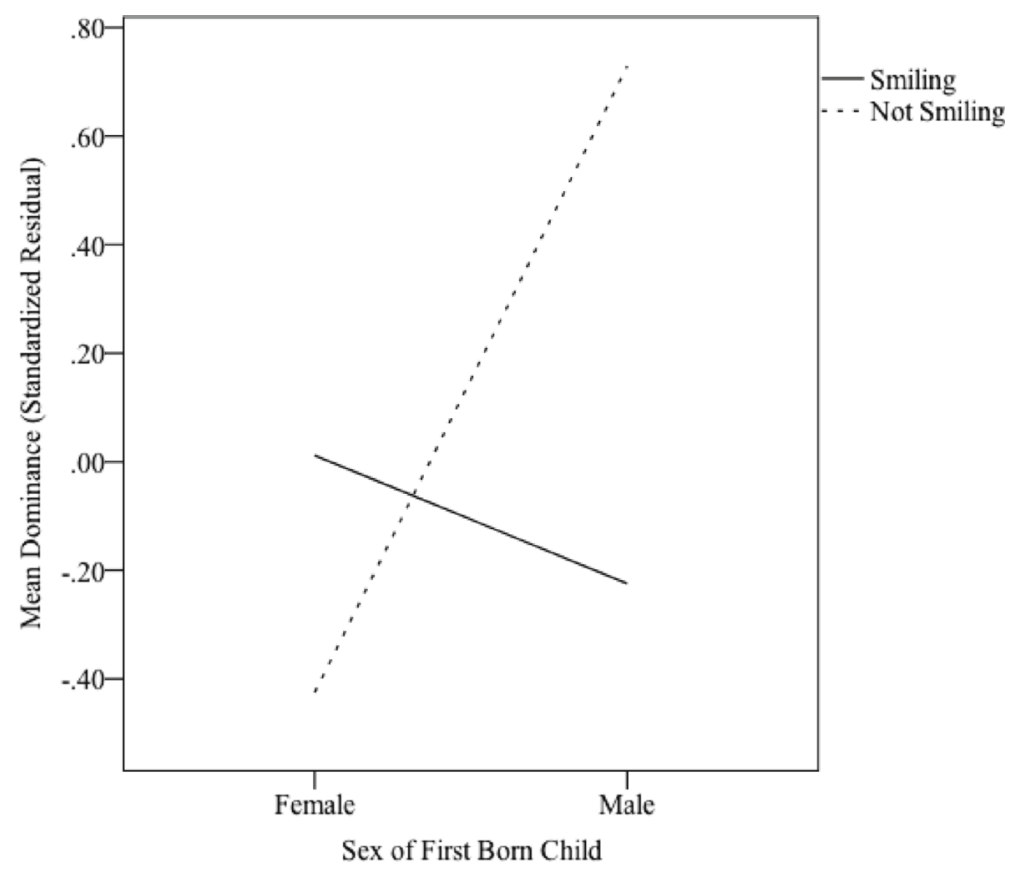

Note: Difference is significant for smiling faces $p<.05$.

Fig. 1 Interaction effect of sex of first-born child and facial expression on ratings of mother's facial dominance 
slope for mother dominance was significant when father dominance was high $(+1 S D)$ $(B(S E)=.65(.31), p=.04)$, but not when it was low $(-1 S D)(p>.05)$. A plot of the probability of a first-born son predicted by mother facial dominance as a function of high $(1 S D)$ and low $(-1 S D)$ father dominance is shown in Fig. 2. These data indicate that when father dominance is high, the probability of a first-born son increases with mother dominance.

Given the interaction between sex of first-born child and facial expression observed for mothers' faces, we then explored the same relationships using the same set of analyses but only with couples where the mother was smiling $(n=$ 90) (the small number of couples $(n=27)$ where the mother not smiling precluded these analyses for this group). Results are shown in Table 4. This time, the first model with mother dominance and father dominance included as predictors approached significance $\left(-2 \mathrm{LL}=116.10, \chi^{2}(2, n=90)=5.23, p=.06\right.$, $\left.R^{2}=.08\right)$ and accurately predicted $78 \%$ of female first-born offspring and $33 \%$ of male first-born offspring, yielding an overall classification rate of $59 \%$. Father dominance was a significant predictor of offspring sex $(B(\mathrm{SE})=-.50$ $(.25), p=.04)$, indicating that as father dominance increases, so does the probability of a first-born daughter. The effect of mother dominance was not significant $(p>.05)$, but it is important to note the reduction in power associated with the limited sample size in this analysis. Adding the interaction between mother dominance and father dominance did not improve the model $(-2 \mathrm{LL}=$ $\left.116.10, \chi^{2}(2, n=90)=5.23, p=.06, R^{2}=.08\right)$.

Table 4 Binary logistic regression analysis using sex of first-born child and facial dominance

\begin{tabular}{|c|c|c|c|}
\hline & $B(S E)$ & Odds Ratio (95 \% CI) & $p$ \\
\hline \multicolumn{4}{|l|}{ All couples $(n=117)$} \\
\hline \multicolumn{4}{|l|}{ Model 1} \\
\hline Mother facial dominance & $.16(.19)$ & $1.17(.80-1.71)$ & .41 \\
\hline Father facial dominance & $-.18(.20)$ & $.83(.57-1.22)$ & .35 \\
\hline \multicolumn{4}{|l|}{ Model 2} \\
\hline Mother facial dominance & $.19(.21)$ & $1.30(.81-1.82)$ & .36 \\
\hline Father facial dominance & $-.27(.23)$ & $.77(.49-1.19)$ & .23 \\
\hline Mother X father facial dominance & $.46(.21)$ & $1.58(1.05-2.39)$ & .03 \\
\hline \multicolumn{4}{|l|}{ Only smiling mothers $(n=90)$} \\
\hline \multicolumn{4}{|l|}{ Model 1} \\
\hline Mother facial dominance & $-.30(.24)$ & $.74(.46-1.17)$ & .19 \\
\hline Father facial dominance & $-.50(.25)$ & $.61(.38-.99)$ & .04 \\
\hline \multicolumn{4}{|l|}{ Model 2} \\
\hline Mother facial dominance & $-.31(.24)$ & $.74(.46-1.18)$ & .21 \\
\hline Father facial dominance & $-.50(.27)$ & $.60(.36-1.02)$ & .06 \\
\hline Mother $\mathrm{X}$ father facial dominance & $.28(.25)$ & $1.33(.82-2.15)$ & .25 \\
\hline
\end{tabular}




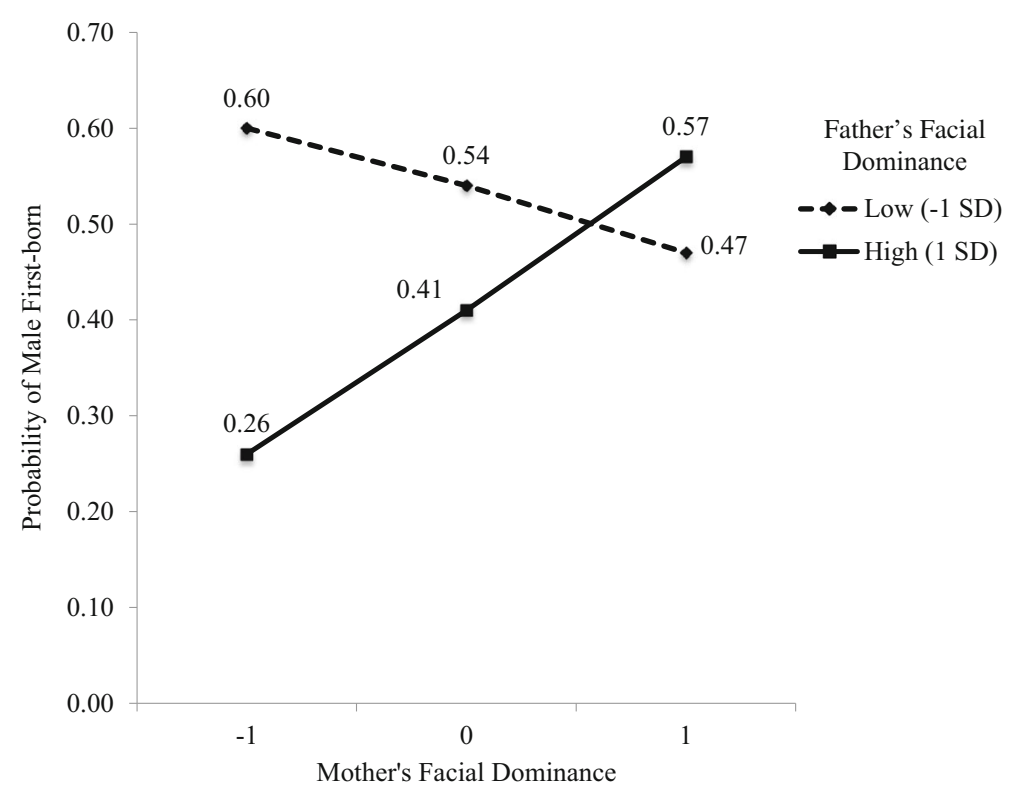

Note: Slope for low father dominance is not significantly different from 0 ; slope for high father dominance is significantly different from $0, p<.05$.

Fig. 2 Probability of a first-born son as a function of mother and father facial dominance, all couples

\section{Parent Behavioral Dominance and Offspring Sex Ratio}

Dominance measures were used to test the hypothesis that parent behavioral dominance has a positive influence on the production of sons. Previous research has shown that mothers and fathers may be more likely to have additional children if their previous ones are all of the same sex, and that this in turn effects the analysis of offspring sex ratios (e.g., Stansfield and Carlton 2007; Gelman 2007). In order to control for this, we again tested whether parent dominance predicts offspring sex in families using the sex of the first-born child as our dependent variable. This approach has been used in other studies of TWH effects in humans (e.g., Kanazawa 2011).

Valid responses were obtained for 132 mothers and 129 fathers. Of these, one mother failed to complete the SD and $\mathrm{AD}$ scales, and one father failed to complete the SAT. These missing values were replaced with the overall means for mothers, and overall mean for fathers, respectively. In order to ensure that behavioral measures were not influenced by pre-existing physiological or mental conditions, we excluded data from an additional 4 mothers (depression, $n=3$; bipolar disorder, $n=1$ ) and 6 fathers (depression, $n=1$; cancer, $n=2$; neurological disorders, $n=3$ ), leaving a total sample of 128 mothers and 123 fathers. Descriptive data obtained from mothers and fathers for the IPIP, SAT, SD, and AD scales are shown in Table 5.

Zero-Order Correlations Scores obtained for mothers and fathers were positively correlated for the SAT $(r=.27, p<.01)$, SD $(r=.21, p=.02)$, and $\mathrm{AD}(r=.24, p<.01)$. 
Table 5 Scores obtained from mothers and fathers on behavioral dominance measures

\begin{tabular}{lll}
\hline & $\begin{array}{l}\text { Mothers }(n=128) \\
\text { Mean (SD) }\end{array}$ & $\begin{array}{l}\text { Fathers }(n=123) \\
\text { Mean (SD) }\end{array}$ \\
\hline Dominance Subscale (IPIP) & $31.16(6.98)$ & $33.78(7.93)$ \\
Simple Adjective Test (SAT) & $3.40(2.45)$ & $4.38(2.80)$ \\
Sociable Dominance Subscale (SD) & $28.46(5.93)$ & $30.85(5.74)$ \\
Aggressive Dominance Subscale (AD) & $18.46(5.36)$ & $20.61(5.50)$ \\
\hline
\end{tabular}

There was no significant relationship between mother and father scores on the IPIP $(r=.07, p>.05)$ (Table 3$)$. For mothers, facial dominance ratings were negatively correlated with scores on the behavioral dominance composite $(r=-.20, p=.03)$ (Table 1). A similar result was obtained when only smiling mothers were considered $(n=90)(r=-.24$, $p=.03$ ), but was no longer significant when only mothers who were not smiling were considered $(n=27)(r=-.17, p=.40)$. No significant correlation was observed for fathers $(r=-.02, p>.05)$ (Table 2), regardless of facial expression $(r s<-.02, p s>.05)$.

Parent Behavioral Dominance as Predictors of Offspring Sex Ratio Since scores obtained for mothers on the IPIP, SAT, SD, and AD were all significantly positively correlated with each other (Table 1), and for fathers, all measures were significantly positively correlated with each other except for the SAT and AD (Table 2), we created composite z-scores for both mother and father dominance behaviors. Mother and father dominance composite $\mathrm{z}$-scores were also positively correlated $(r=.28, p<.01)$.

Mothers who had a son first (Mean $\pm \mathrm{SD}=.19 \pm .85$ ) scored significantly higher in dominance than mothers who had a daughter first $(\mathrm{Mean} \pm \mathrm{SD}=-.17 \pm 1.10)(t(122.61)=$ $-2.06, p=.04$ ). Similarly, fathers who had a son first (Mean $\pm \mathrm{SD}=.23 \pm .96)$ scored significantly higher in dominance than fathers who had a daughter first (Mean $\pm \mathrm{SD}=$ $-.21 \pm .99)(t(121)=-2.51, p=.01)$.

When mother dominance behavior and father dominance behavior were entered into a binary logistic regression as predictors of sex of first-born child, the model was significant $\left(-2 \mathrm{LL}=158.29, \chi^{2}(2, n=121)=9.24, p=.01, R^{2}=.10\right)$. The model accurately predicted $60 \%$ of female first-born offspring and $52 \%$ of male first-born offspring, yielding an overall classification rate of $56 \%$. Adding the interaction between mother and father dominance behavior as a predictor did not improve the model $(-2 \mathrm{LL}=$ 157.78, $\left.\chi^{2}(2, n=121)=.51, p=.02, R^{2}=.10\right)$. Results obtained from these two models are given in Table 6 .

\section{Discussion}

Our results provide partial support for the hypothesis that women select mates who demonstrate heritable qualities that confer sex-specific reproductive advantages on their future offspring. We found significant correlations between parent facial attractiveness and dominance ratings, and between their self-reported dominance characteristics. In 
Table 6 Binary logistic regression analysis using sex of first-born child and behavioral dominance

\begin{tabular}{llll}
\hline & $B(S E)$ & Odds ratio $(95 \% \mathrm{CI})$ & $p$ \\
\hline $\begin{array}{l}\text { All couples }(n=121) \\
\text { Model 1 }\end{array}$ & & & \\
$\quad$ Mother composite dominance & $.31(.20)$ & $1.36(.92-2.01)$ & .12 \\
$\quad$ Father composite dominance & $.42(.21)$ & $1.52(1.02-2.28)$ & .04 \\
Model 2 & & & .14 \\
$\quad$ Mother composite dominance & $.30(.20)$ & $1.35(.91-1.99)$ & .04 \\
$\quad$ Father composite dominance & $.44(.21)$ & $1.55(1.02-2.33)$ & .48 \\
$\quad$ Mother X father composite dominance & $-.15(.21)$ & $.87(.58-1.30)$ & \\
\hline
\end{tabular}

addition, we found evidence for a positive effect of mothers' facial dominance on the probability of a first-born son, but only when fathers' facial dominance was high. Specifically, we found that when paired with fathers whose faces were rated as high in dominance, mothers whose faces were rated as being low in dominance were more likely to conceive a daughter. Mother dominance had no influence on offspring sex when father dominance was low. Although the effect of mothers' facial dominance was no longer significant when faces that were not smiling were excluded, higher father facial dominance continued to increase the probability of a daughter. We also found that dominance behavior in mothers and fathers positively predicted the probability of a first-born son. Taken together, our results suggest that women's mate selection is influenced by men's dominance, and that this is related to the sex of their future offspring; however, there may be other factors used in concert with dominance in assessment of male mate quality.

We found an interaction between mother and father facial dominance that approached significance, suggesting that low mother dominance is associated with an increased likelihood of female offspring, but only when father dominance is high. Furthermore, after controlling for mothers' facial expression, father dominance became the main predictor of offspring sex. Thus, male dominance appears to be attractive to both mothers of sons and mothers of daughters, despite the assumption that heritable physical characteristics of dominance are more beneficial to sons. Although still consistent with $\mathrm{TWH}$, this result is contrary to the hypothesis that mother dominance should increase the probability of conceiving a son. The exact reason for this finding is unclear, but one possible reason is that we were unable to fully detect mothers' facial dominance due to their facial expressions. Smiling women were rated significantly lower in dominance than non-smiling women, making it crucial that expression is controlled in future studies.

Another possible explanation for the contrast between our results for faces and the TWH is that selection for attractiveness in female mates is stronger than that of dominance. Although it can be beneficial for intrasexual interactions, males do not exhibit clear preferences for female dominance (reviewed in Campbell 1999). Female attractiveness and femininity are both highly valued by males, who report even stronger preferences for a feminine face when their testosterone is higher (Welling et al. 2008). More dominant men might prefer more attractive, and less dominant, women as long- 
term mates, resulting in a bias toward lower offspring sex ratios. Buss and Shackelford (2008) showed that the most attractive women in their sample had the strongest preferences for male indicators of parent quality, financial investment, and physical attractiveness, suggesting that their own mate value allowed them greater selection in obtaining the ideal male mate. Future studies investigating sexual selection and offspring sex ratios should include these possible indicators of male and female mate value in order to assess their relative contribution to long-term mate choice.

Lastly, given that our sample was comprised of parents of undergraduate students, the majority of whom were middle to upper-middle class, engaged in long-term, committed relationships, and only had 1-2 children, it is possible that we have failed to measure the full range of dominance characteristics in male and female mates. Dominant and masculine looking males are perceived to exhibit less positive qualities, such as warmth, honesty, and willingness to invest in parenting (Perrett et al. 1998). In addition, Booth and Dabbs (1993) reported that men with higher testosterone were less likely to marry, and when they did, they were more likely to divorce and to have had extramarital partners. Thus, it is possible that the most dominant males, who may be most likely to produce sons, were not adequately represented in our study. Future work should attempt to replicate our findings with males and females prior to mating as well as in different cultural and socioeconomic circumstances.

Our results suggest that dominance behavior is also related to mate selection in accordance with offspring sex. Although our behavioral questionnaires were collected several years after parents had finished having children and were thus less sensitive to detecting a direct influence on offspring sex as they would have been during reproduction, we did observe a positive effect of parent dominance on the probability of a first-born son. This suggests that dominant mothers might prefer dominant fathers. It could be argued that parental dominance was the result rather than the cause of having a son compared to a daughter, but this is unlikely given the relationships that we also observed between offspring sex and facial dominance characteristics obtained from early photographs. In addition, although state dominance might be expected to vary throughout the years in our sample, levels of trait dominance should remain relatively stable and thus provide a proxy for dominance at the time of conception.

Lastly, we found no evidence that the face is an effective prospective indicator of offspring sex. Objective raters were not better than chance at predicting offspring sex from faces. We did not provide raters with any explanation or description of this task other than to ask them what sex of child they predicted the depicted individual would have as their first-born child, and so it is possible that participants held pre-existing beliefs that the determination of an individual's sex is simply a product of chance (i.e., equal probability of an egg fusing with an X- or Y-chromosome bearing sperm), and answered with little thought. Their detection of differences between masculinity, attractiveness, and dominance in parents of sons and mothers of daughters suggests that they may actually have some ability to detect offspring sex from faces. Thus, the inclusion of more directive instructions might be helpful in making this determination in future studies.

Some final limitations of our study are worth noting. First, the retrospective nature of our study design limited our ability to control the photographs used for parent ratings. In particular, we were unable to completely control for facial expression. Although we attempted to statistically control for these effects, reduced sample size and subsequent 
power in binary logistic regression analyses limits the generalizability of these findings. Nonetheless, facial expression was shown to influence our findings and future studies should seek to elicit photographs where participants are not smiling. Additional factors such as ethnicity, make-up, glasses, facial hair, and other adornments were also not controlled for, and may have altered the judgments made by participants. Second, although our raters were naïve to the study hypothesis when they made judgments about parent faces, it is possible that the nature of the characteristics we asked them to rate may have influenced their predictions about the sexes of individual's offspring. However, our finding that raters were not better able than chance to predict a person's offspring sex indicates that their ratings were unrelated to any knowledge or bias related to ideas about dominance and the sexes of children that parents might have. Future work might seek to obtain photographs using a more controlled method. Third, we were not able to assess the possibility of extra-pair copulations and subsequent genetic relationships between fathers and offspring, nor did we assess the sexes of any offspring lost to miscarriage or stillbirth, the latter of which would provide additional information about manipulation of primary offspring sex ratios. These factors should be taken into consideration in future research.

In summary, the present study provides an initial demonstration that when mated to high dominance men, women rated lower in facial dominance may be more likely to have a daughter as a first-born child than women rated higher in facial dominance. In addition, parents who are more behaviorally dominant are more likely to produce firstborn sons. These results provide partial support for the hypothesis that the assessment of men's long-term mate value may be influenced by women's internal cues signaling the probability of male versus female future offspring, resulting in preferences for males demonstrating heritable traits of sex-specific reproductive benefit to offspring and thereby enhancing the inclusive fitness of the mother. Future research should identify additional factors that influence women's assessments of mate value, and how these are related to her future offspring sex.

Author Note The authors wish to thank E. Chakraborti for her assistance with data collection. This research was funded by Discovery Grant 0194522 from the Natural Sciences and Engineering Research Council of Canada (NSERC) to N. V. Watson.

\section{References}

Akande, A. (1999). Maternal dominance and the sex of a baby. Early Child Development and Care. doi:10. $1080 / 0300443991520107$.

Booth, A., \& Dabbs, J. M. (1993). Testosterone and men's marriages. Social Forces, 72, 463-477.

Boothroyd, L. G., Jones, B. C., Burt, D. M., \& Perrett, D. I. (2007). Partner characteristics associated with masculinity, health and maturity in faces. Personality and Individual Differences. doi:10.1016/j.paid. 2007.03.008.

Brown, G. R. (2001). Sex-biased investment in nonhuman primates: can Trivers \& Willard's theory be tested? Animal Behavior. doi:10.1006/anbe.2000.1659.

Burriss, R. P., Welling, L. L. M., \& Puts, D. A. (2011). Mate-preferences drives mate-choice: men's self-rated masculinity predicts their female partner's preference for masculinity. Personality and Individual Differences. doi:10.1016/j.paid.2011.08.018. 
Buss, D. M. (2007). The evolution of human mating. Acta Psychologica Sinica, 39, 502-512.

Buss, D. M., \& Shackelford, T. K. (2008). Attractive women want it all: good genes, economic investment, parenting proclivities, and emotional commitment. Evolutionary Psychology, 6, 134-146.

Cameron, E. Z., \& Dalerum, F. (2009). A Trivers-Willard effect in contemporary humans: male-biased sex ratios among billionaires. PLOS One, 4, 1-4. doi:10.1371/journal.pone.0004195.

Campbell, A. (1999). Staying alive: evolution, culture, and women's intrasexual aggression. Behavioral and Brain Sciences, 22, 203-252.

Carré, J. M., \& McCormick, C. M. (2008). In your face: facial metrics predict aggressive behaviour in the laboratory and in varsity and professional hockey players. Proceedings of the Royal Society of London B: Biological Sciences, 275(1651), 2651-2656.

Cashdan, E. (1998). Women's mating strategies. Evolutionary Anthropology, 5, 134-143.

Central Intelligence Agency. (2013). Sex ratio. In The World Factbook. Retrieved from https://www.cia.gov/ library/publications/the-world-factbook/fields/2018.html

Clutton-Brock, T. H., \& Iason, G. R. (1986). Sex ratio variation in mammals. Quarterly Review of Biology, 61, 339-374.

Cornwell, R. E., \& Perrett, D. I. (2008). Sexy sons and sexy daughters: the influence of parents' facial characteristics on offspring. Animal Behavior. doi:10.1016/j.anbehav.2008.07.031.

Dahl, G. B., \& Moretti, E. (2008). The demand for sons. Review of Economic Studies, 75, 1085-1120.

Dawson, J. F. (2014). Moderation in management research: what, why, when, and how. Journal of Business Psychology. doi:10.1007/s10869-013-9308-7.

DeBruine, L. M., Jones, B. C., Little, A. C., Boothroyd, L. G., Perrett, D. I., Penton-Voak, I. S., et al. (2006). Correlated preferences for facial masculinity and ideal or actual partner's masculinity. Proceedings of the Royal Society B: Biological Sciences. doi:10.1098/rspb.2005.3445.

Fink, B., Neave, N., \& Seydel, H. (2007). Male facial appearance signals physical strength to women. American Journal of Human Biology. doi:10.1002/ajhb.20583.

Gelman, A. (2007). Letter to the editor regarding some papers of Dr. Satoshi Kanazawa. Journal of Theoretical Biology. doi:10.1016/j.jtbi.2006.11.005.

Geniole, S. N., Keyes, A. E., Mondloch, C. J., Carre, J. M., \& McCormick, C. M. (2012). Facing aggression: cues differ for female versus male faces. PLOS One, 7(1), 1-12.

Gibson, M. A., \& Mace, R. (2003). Strong mothers bear more sons in rural Ethiopia. Proceedings of the Royal Society B. doi:10.1098/rbsl.2003.0031.

Goldberg, L. R., Johnson, J. A., Eber, H. W., Hogan, R., Ashton, M. C., Cloninger, C. R., et al. (2006). The international personality item pool and the future of public-domain personality measures. Journal of Research in Personality. doi:10.1016/j.jrp.2005.08.007.

Grant, V. J. (1990). Maternal personality and sex of infant. British Journal of Medical Psychology, 63, 261266.

Grant, V. J. (1994). Maternal dominance and the conception of sons. British Journal of Medical Psychology, $67,343-351$.

Grant, V. J. (1998). Maternal personality, evolution and the sex ratio. New York: Routledge.

Grant, V. J., \& France, J. T. (2001). Dominance and testosterone in women. Biological Psychology. doi:10. 1016/S0301-0511(01)00100-4.

Grant, V. J., \& Irwin, R. J. (2005). Follicular fluid steroid levels and subsequent sex of bovine embryos. Journal of Experimental Zoology. doi:10.1002/jez.a.233.

Hank, K. (2007). Parental gender preferences and reproductive behavior: a review of the recent literature. Journal of Biosocial Science, 39, 759-767. doi:10.1017/S0021932006001787.

Hesketh, T., \& Xing, Z. W. (2006). Abnormal sex ratios in human populations: causes and consequences. PNAS, 103, 229-234. doi:10.1073/pnas.0602203103.

Hewison, A. J. M., \& Gaillard, J. (1999). Successful sons or advantaged daughters? The Trivers-Willard model and sex-biased maternal investment in ungulates. Trends in Evolution and Ecology, 14, 229-234. doi:10.1016/S0169-5347(99)01592-X.

Hiraiwa-Hasegawa, M. (1993). Skewed birth sex ratios in primates: should high-ranking mothers have daughters or sons? Trends in Evolution and Ecology. doi:10.1016/0169-5347(93)90040-V.

James, W. H. (1987). The human sex ratio. Part 2: a hypothesis and a program of research. Human Biology, 59, 873-900.

James, W. H. (1996). Evidence that mammalian sex ratios at birth are partially controlled by parental hormones at the time of conception. Journal of Theoretical Biology, 180, 271-286.

James, W. H. (2004). Further evidence that mammalian sex ratios at birth are partially controlled by parental hormones around the time of conception. Human Reproduction, 19, 1250-1256. doi:10.1093/humrep/ deh245. 
James, W. H. (2009). The variations of human sex ratio at birth during and after wars, and their potential explanations. Journal of Theoretical Biology, 257, 116-123. doi:10.1016/j.jtbi.2008.09.028.

James, W. H. (2010). Behavioural and biological determinants of human sex ratio at birth. Journal of Biosocial Science, 42, 587-599. doi:10.1017/S002193201000012X.

Johnston, V. S., Hagel, R., Franklin, M., Fink, B., \& Grammer, K. (2001). Male facial attractiveness: evidence for hormone-mediated adaptive design. Evolution and Human Behavior. doi:10.1016/S1090-5138(01) 00066-6.

Kalma, A. P., Visser, L., \& Peeters, A. (1993). Sociable and aggressive dominance: personality differences in leadership style? Leadership Quarterly. doi:10.1016/1048-9843(93)90003-C.

Kanazawa, S. (2005). Big and tall parents have more sons: further generalizations of the Trivers-Willard hypothesis. Journal of Theoretical Biology. doi:10.1016/j.jtbi.2005.02.010.

Kanazawa, S. (2008). Battered women have more sons: a possible evolutionary reason why some battered women stay. Journal of Evolutionary Psychology. doi:10.1556/JEP.2008.1007.

Kanazawa, S. (2011). Beautiful British parents have more daughters. Reproductive Sciences. doi:10.1177/ 1933719110393031.

Kanazawa, S., \& Reyniers, D. J. (2009). The role of height in the sex difference in intelligence. American Journal of Psychology, 122, 527-536.

Lefevre, C. E., Etchells, P. J., Howell, E. C., Clark, A. P., \& Penton-Voak, I. S. (2014). Facial width-to-height ratio predicts self-reported dominance and aggression in males and females, but a measure of masculinity does not. Biology Letters. doi:10.1098/rsbl.2014.0729.

Manning, J. T., \& Quinton, S. (2007). Association of digit ratio (2D:4D) with self-reported attractiveness in men and women. Journal of Individual Differences, 28, 73-77. doi:10.1027/1614-0001.28.2.73.

Manning, J. T., Anderton, R., \& Washington, S. M. (1996). Women's waists and the sex ratio of their progeny: evolutionary aspects of the ideal female body shape. Journal of Human Evolution, 31, 41-47.

Manning, J. T., Martin, S., Trivers, R. L., \& Soler, M. (2002). 2nd to 4th digit ratio and offspring sex ratio. Journal of Theoretical Biology, 217, 93-95. doi:10.1027/jtbi.2002.3014.

Marleau, J. D., \& Saucier, J. (2002). Preferences for a first-born boy in western societies. Journal of Biosocial Science, 34, 13-27.

Mueller, U., \& Mazur, A. (1997). Facial dominance in Homo sapiens as honest signaling of male quality. Behavioral Ecology, 8, 569-579.

Neave, N., Laing, S., Fink, B., \& Manning, J. T. (2003). Second to fourth digit ratio, testosterone, and perceived male dominance. Proceedings of the Royal Society B: Biological Sciences, 270, 2167-2172. doi:10.1098/rspb.2003.2502.

Özener, B. (2012). Facial width-to-height ratio in a Turkish population is not sexually dimorphic and is unrelated to aggressive behavior. Evolution and Human Behavior, 33(3), 169-173. doi:10.1016/j. evolhumbehav.2011.08.001.

Pawlowski, B., \& Jasienska, G. (2008). Women's body morphology and preferences for sexual partner's characteristics. Evolution and Human Behavior. doi:10.1016/j.evolhumbehav.2007.07.003.

Perrett, D. I., Lee, K. J., Penton-Voak, I., Rowland, D., Yoshikawa, S., Burt, D. M., et al. (1998). Effects of sexual dimorphism on facial attractiveness. Nature. doi:10.1038/29772.

Puts, D. A. (2010). Beauty and the beast: mechanisms of sexual selection in humans. Evolution and Human Behavior. doi:10.1016/j.evolhumbehav.2010.02.005.

Puts, D. A., Jones, B. C., \& DeBruine, L. M. (2012). Sexual selection on human faces and voices. Journal of Sex Research. doi:10.1080/00224499.2012.658924.

Quist, M. C., Watkins, C. D., Smith, F. G., DeBruine, L. M., \& Jones, B. C. (2011). Facial masculinity is a cue to women's dominance. Personality and Individual Differences, 50(7), 1089-1093. doi:10.1016/j.paid. 2011.01.032.

Ray, J. G., Henry, D. A., \& Urquia, M. L. (2012). Sex ratios among Canadian liveborn infants of mothers from different countries. Canadian Medical Association Journal, 184, E492-E496.

Rhodes, G. (2006). The evolutionary psychology of facial beauty. Annual Review of Psychology, 57, 199-226.

Rhodes, G., Hickford, C., \& Jeffery, L. (2000). Sex-typicality and attractiveness: are supermale and superfemale faces super-attractive? British Journal of Psychology, 91, 125-140.

Sadalla, E. K., Kenrick, D. T., \& Vershure, B. (1987). Dominance and heterosexual attraction. Journal of Personality and Social Psychology, 52, 730-738.

Scott, I. M. L., Pound, N., Stephen, I. D., Clark, A. P., \& Penton-Voak, I. S. (2010). Does masculinity matter? The contribution of masculine face shape to male attractiveness in humans. PloS One, 5, 1-10.

Sell, A., Cosmides, L., Tooby, J., Sznycer, D., von Rueden, C., \& Gurven, M. (2009). Human adaptations for the visual assessment of strength and fighting ability from the body and face. Proceedings of the Royal Society of London B: Biological Sciences, 276, 575-584. doi:10.1098/rspb.2008.1177. 
Sheldon, B. C., \& West, S. A. (2004). Maternal dominance, maternal condition, and offspring sex ratio in ungulate animals. American Naturalist, 163, 40-54.

Singh, D. (1994). Is thin really beautiful and good? Relationship between waist-to-hip ratio (WHR) and female attractiveness. Personality and Individual Differences. doi:10.1016/0191-8869(94)90116-3.

Singh, D., \& Zambarno, R. J. (1997). Offspring sex ratio in women with android body fat distribution. Human Biology, 69, 545-556.

Stanislaw, H., \& Todorov, N. (1999). Calculation of signal detection theory measures. Behavior Research Methods, Instruments, \& Computers. doi:10.3758/BF03207704.

Stansfield, W. D., \& Carlton, M. A. (2007). Human sex ratios and sex distribution in sibships of size 2. Human Biology, 79, 255-260.

Trebicky, V., Havlicek, J., Roberts, S. C., Little, A. C., \& Kleisner, K. (2013). Perceived aggressiveness predicts fighting performance in mixed-martial-arts fighters. Psychological Science, 24(9), 1664-1672. doi:10.1177/0956797613477117.

Trivers, R. L. (1972). Parental investment and sexual selection. In B. Campbell (Ed.), Sexual selection and the descent of man, 1871-1971 (pp. 136-179). Chicago, IL: Aldine.

Trivers, R. L., \& Willard, D. E. (1973). Natural selection of parental ability to vary the sex ratio of offspring. Science, 179, 90-92.

Welling, L. L. M., Jones, B. C., DeBruine, L. M., Conway, C. A., Law Smith, M. J., Little, A. C., et al. (2007). Raised salivary testosterone in women is associated with increased attraction to masculine faces. Hormones and Behavior. doi:10.1016/j.yhbeh.2007.01.010.

Welling, L. L. M., Jones, B. C., DeBruine, L. M., Smith, F. G., Feinberg, D. R., Little, A. C., et al. (2008). Men report stronger attraction to femininity in women's faces when their testosterone levels are high. Hormones and Behavior. doi:10.1016/j.yhbeh.2008.07.012.

Zilioli, S., Sell, A. N., Stirrat, M., Jagore, J., Vickerman, W., \& Watson, N. V. (2014). Face of a Fighter: Bizygomatic width as a Cue of Formidability. Aggressive Behavior, 9999, 1-9. doi:10.1002/ab.21544. 